\title{
Hybrid TE-TM scheme for time domain numerical calculations of wakefields in structures with walls of finite conductivity
}

\author{
Andranik Tsakanian \\ CANDLE, Acharyan 31, 0040, Yerevan, Armenia \\ Martin Dohlus and Igor Zagorodnov \\ DESY, Notkestrasse 85, 22607, Hamburg, Germany \\ (Received 23 February 2011; published 9 May 2012)
}

\begin{abstract}
In particle accelerators a preferred direction, the direction of motion, is well defined. If in a numerical calculation the (numerical) dispersion in this direction is suppressed, a quite coarse mesh and moderate computational resources can be used to reach accurate results even for extremely short electron bunches. Several approaches have been proposed in the past decades to reduce the accumulated dispersion error in wakefield calculations for perfectly conducting structures. In this paper we extend the TE/TM splitting algorithm to a new hybrid scheme that allows for wakefield calculations in structures with walls of finite conductivity. The conductive boundary is modeled by one-dimensional wires connected to each boundary cell. A good agreement of the numerical simulations with analytical results and other numerical approaches is obtained.
\end{abstract}

DOI: 10.1103/PhysRevSTAB.15.054401

PACS numbers: 29.27.Bd, 02.60.Cb, 02.70.Bf

\section{INTRODUCTION}

Preservation of very small phase space volume (emittance) of electron bunches is one of the challenges in modern linear accelerators for fundamental and applied research [1-3]. During acceleration the bunch interacts with the surrounding structure and excites electromagnetic fields that act back on the bunch. These fields, known as wakefields, affect the motion of particles, spoil the bunch quality, and can lead to beam instabilities and subsequent beam loss. The effects of wakefields on the bunch can be described by longitudinal and transverse wake potentials that represent the integrated Lorenz force in longitudinal and transverse directions acting on a trailing test particle.

Computation of wake potentials is one of the important problems in particle accelerator physics [4,5] Analytical solutions (see, for example, [4-8]) have been obtained for a number of relatively simple geometries and are important for understanding the properties of wakefields. Nevertheless, the only practical way of calculating and studying the electromagnetic fields in complicated threedimensional (3D) structures is the application of numerical methods.

A self-consistent treatment of the beam dynamics can be done with a particle-in-cell (PIC) code [9]. The electromagnetic fields in many PIC codes are computed using the finite difference time domain (FDTD) method [10,11]. As any numerical mesh approach, the conventional FDTD

Published by the American Physical Society under the terms of the Creative Commons Attribution 3.0 License. Further distribution of this work must maintain attribution to the author(s) and the published article's title, journal citation, and DOI. scheme [10] used in MAFIA [12], TBCI [13], and other wake and PIC codes suffers from numerical grid dispersion, i.e., the phase velocity of the numerical waves is slower than the physical one. Hence, high energy particles can travel in vacuum faster than their own radiation. This effect is commonly referred to as numerical Cherenkov radiation [14], which due to its accumulative character corrupts the simulation.

More complications arise for structures with walls of finite conductivity, since modeling of electromagnetic wave propagation in a conductor requires a much more dense mesh than modeling of wave propagation in vacuum. Several numerical codes have been developed to solve wakefield problems in frequency and time domains [12,13,15-17] for perfectly conducting structures. From the existing numerical codes only MAFIA and CST MICROWAVE STUDIO [15] can model structures with finite resistivity but the algorithms used suffer from numerical dispersion and are inefficient for very short electron bunches. To avoid the errors due to numerical dispersion in the longitudinal direction, a TE/TM splitting algorithm has been proposed, for example, in [16].

In this paper a new (longitudinally) dispersion-free hybrid numerical scheme is described which has been developed to evaluate the wakefields in structures with walls of finite conductivity. The conductive boundary is modeled in this scheme by one-dimensional wires connected to each boundary cell. The stability and the convergence of the new numerical scheme are studied. A good agreement of the numerical simulations with analytical results and other numerical approaches has been obtained. The new scheme allows the calculation of the wakefields excited by an ultrarelativistic charge in 3D structures of arbitrarily 
shaped geometries with walls of finite high conductivity. However, we have realized the scheme only for rotationally symmetric structures and will describe here only the scheme for this case.

The paper is structured in the following way. In Secs. II, III, and IV the formulation of the problem and an introduction to space and time discretization of Maxwell's equations are given. In Sec. V a one-dimensional (1D) numerical scheme for time domain modeling of conductive materials is introduced. In Sec. VI a new hybrid TE/TM implicit time domain numerical scheme for rotationally symmetric structures with walls of finite conductivity is presented. In Sec. VII the convergence of the new hybrid scheme is studied. Finally, in Sec. VIII a number of numerical tests and a comparison with existing solutions are given.

\section{FORMULATION OF THE PROBLEM}

Consider an ultrarelativistic electron bunch with rigid longitudinal distribution $\rho$, moving along a structure with the speed of light $c$ (Fig. 1). The internal region of the structure $\Omega$ is bounded by the resistive infinite wall with conductivity $\kappa$.

We are looking for electromagnetic fields $\vec{E}, \vec{H}$ induced by the bunch due to its interaction with the surrounding structure. In order to find these fields we need to solve the Maxwell equations,

$$
\begin{aligned}
\vec{\nabla} \times \vec{H} & =\vec{j}+\frac{\partial \vec{D}}{\partial t}, \quad \vec{\nabla} \times \vec{E}=-\frac{\partial \vec{B}}{\partial t}, \quad \vec{\nabla} \cdot \vec{D}=\rho, \\
\vec{\nabla} \cdot \vec{B} & =0, \quad \vec{D}=\varepsilon \vec{E}, \quad \vec{B}=\mu \vec{H},
\end{aligned}
$$

with the initial conditions

$$
\vec{E}(t=0)=\vec{E}_{0}, \quad \vec{H}(t=0)=\vec{H}_{0},
$$

where $t$ is the time, $\vec{j}=\vec{j}_{b}+\vec{j}_{c}$ is the current density, $\vec{j}_{b}=\vec{e}_{z} c \rho$ is the charge current, $\vec{j}_{c}=\kappa \vec{E}$ is the current induced in the wall, and $\varepsilon, \mu$ are the dielectric permittivity and the magnetic permeability, respectively. The interface conditions for electromagnetic fields are given by the continuity of tangential components of electric and magnetic fields at the boundary between the vacuum and the conductive wall.

In accelerator applications, the structures are usually supplied by an ingoing pipe and the well-known analytical solution for ultrarelativistic beam in a perfectly conducting

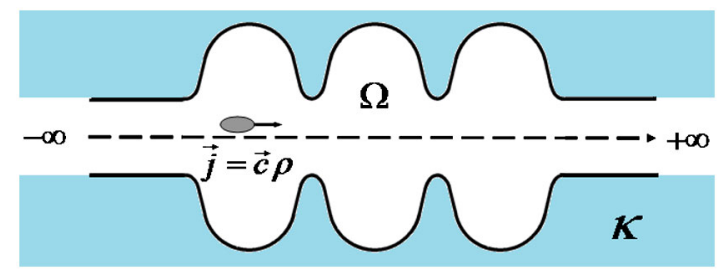

FIG. 1. Charged particle bunch moving through an accelerating structure supplied with infinite pipes. cylindrical pipe [7] can be used as initial field. In general, if the ingoing pipe is not cylindrical or not perfectly conducting the initial field can be found numerically.

In order to solve the system of Eq. (1) we will develop in the next sections a finite difference method. In this paper the boundaries (or interfaces) are modeled by a staircase approximation. The extension of the scheme to include a conformal modeling of the resistive walls will be described in other publications.

\section{SPATIAL DISCRETIZATION AND MAXWELL GRID EQUATIONS}

In this section we describe a spatial discretization of the system (1) in a vacuum region assuming that the field components at the interface to the conductive wall are known a priori.

Following the matrix notations of the finite integration technique (FIT) [18], the continuous problem (1) can be approximated by time-continuous matrix equations on a grid doublet (see Fig. 2):

$$
C \hat{e}=-\frac{d}{d t} \hat{\hat{b}}, \quad C^{T} \hat{h}=\frac{d}{d t} \hat{\hat{d}}+\hat{\hat{j}}, \quad \tilde{S} \hat{\hat{d}}=\hat{q}, \quad S \hat{\hat{b}}=0,
$$

completed by the discrete form of the material relations (constitutive equations)

$$
\hat{e}=M_{\varepsilon^{-1}} \hat{\hat{d}}, \quad \hat{h}=M_{\mu^{-1}} \hat{\hat{b}},
$$

with the discrete inverse permittivity matrix $M_{\varepsilon^{-1}}=$ $\operatorname{diag}\left(M_{\varepsilon_{x}^{-1}}, M_{\varepsilon_{y}^{-1}}, M_{\varepsilon_{z}^{-1}}\right)$ and the inverse permeability ma$\operatorname{trix} M_{\mu^{-1}}=\operatorname{diag}\left(M_{\mu_{x}^{-1}}, M_{\mu_{y}^{-1}}, M_{\mu_{z}^{-1}}\right)$. The material matrices are assumed to be real and symmetric.

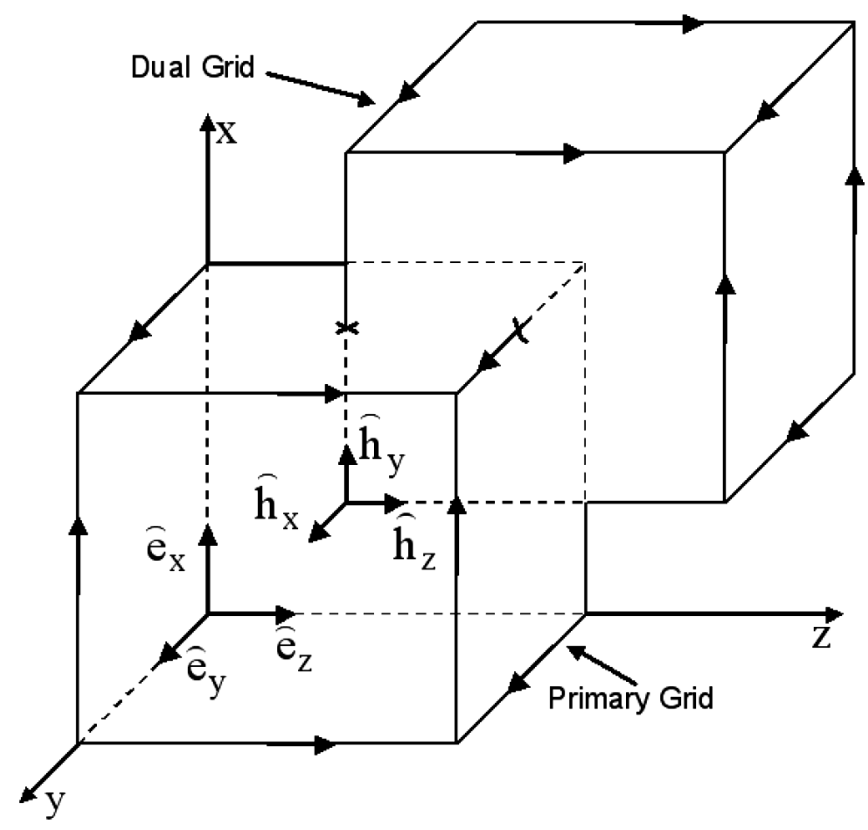

FIG. 2. The allocation of electric and magnetic field components on the grid doublet. 
On a Cartesian $\{x, y, z\}$ grid (Fig. 2) with an appropriate indexing scheme, the curl and divergence matrices can be written in the form

$$
\begin{gathered}
C=\left(\begin{array}{ccc}
0 & -P_{z} & P_{y} \\
P_{z} & 0 & -P_{x} \\
-P_{y} & P_{x} & 0
\end{array}\right), \quad S=\left(\begin{array}{lll}
P_{x} & P_{y} & P_{z}
\end{array}\right), \\
\tilde{S}=\left(\begin{array}{ccc}
-P_{x}^{T} & -P_{y}^{T} & -P_{z}^{T}
\end{array}\right),
\end{gathered}
$$

where the topological matrices $P_{\{x, y, z\}}$ play the role of discrete partial differential operators [18].

Introducing the variables

$$
\begin{aligned}
& \tau=c t, \quad e=\frac{1}{Z_{0}} M_{\varepsilon^{-1}}^{-1 / 2} \hat{e}, \quad h=M_{\mu^{-1}}^{-1 / 2} \hat{h}, \\
& j=M_{\varepsilon^{-1}}^{1 / 2} \hat{\hat{j}}, \quad q=c^{-1} M_{\varepsilon^{-1}}^{1 / 2} \hat{q},
\end{aligned}
$$

where $Z_{0}$ is the free space impedance, the system (2) can be reduced to the skew-symmetric system,

$\frac{d}{d \tau} e=C_{0}^{T} h+j, \quad \frac{d}{d \tau} h=-C_{0} e, \quad S_{h} h=0, \quad S_{e} e=q$,

with the discrete "curl" and "div" operators

$$
\begin{aligned}
& C_{0}=M_{\mu^{-1}}^{1 / 2} C M_{\varepsilon^{-1}}^{1 / 2} \equiv\left(\begin{array}{ccc}
0 & -P_{z}^{0} & P_{y}^{0} \\
P_{z}^{1} & 0 & -P_{x}^{0} \\
-P_{y}^{1} & P_{x}^{1} & 0
\end{array}\right), \\
& S_{e}=\left(-P_{x}^{T} M_{\varepsilon_{x}^{-1}}^{1 / 2}-P_{y}^{T} M_{\varepsilon_{y}^{-1}}^{1 / 2}-P_{z}^{T} M_{\varepsilon_{z}^{-1}}^{1 / 2}\right), \\
& S_{h}=\left(P_{x} M_{\mu_{x}^{-1}}^{1 / 2} P_{y} M_{\mu_{y}^{-1}}^{1 / 2} P_{z} M_{\mu_{z}^{-1}}^{1 / 2}\right) .
\end{aligned}
$$

The equation system (4) is a time-continuous and spacediscrete approximation of the problem (1).

An important feature of the Maxwell grid equations (MGE) when compared with other numerical methods for solving EM field problems is that this set of matrix equations is a consistent discrete representation of the original field equations, i.e., the basic properties of the analytical fields are maintained during the transformation of the continuous problem to the discrete space $[18,19]$.

In the MGE representation (4) a discrete analogue to the analytical equation div curl $=0$ holds:

$$
S_{h} C_{0}=S_{e} C_{0}^{T}=0 .
$$

From this property of the discrete operators follow the discrete charge $q$ and discrete energy $W$ conservation in the time-continuous numerical scheme (4)

$$
\begin{aligned}
\frac{d}{d \tau} q+S_{e} j & =0, \quad \frac{d}{d \tau} S_{h} h=0, \quad \frac{d}{d \tau} W=-\langle e, j\rangle, \\
W(\tau) & =\frac{e^{T}(\tau) e(\tau)+h^{T}(\tau) h(\tau)}{2} .
\end{aligned}
$$

Note that the discrete energy $W$ corresponds to the physical energy of the continuous electromagnetic fields given by $0.5 \int_{V}\left(\varepsilon|E|^{2}+\mu^{-1}|B|^{2}\right) d v$.

\section{TIME DISCRETIZATION}

In this section the discretization of the MGE (4) in time is considered. The field components can be split in time and the "leapfrog" scheme can be applied. Two kinds of splitting ("electric magnetic" and "transverse electric transverse magnetic") are considered.

\section{A. Explicit FDTD method based on "electric-magnetic" splitting of the field components in time}

An explicit scheme has been suggested by Yee [10] where splitting of electric and magnetic field components in time have been used (E/M splitting). On the time axis the electric field components are defined at the same time while the magnetic components are shifted by a half-time step relative to the electric ones. This numerical scheme in matrix form reads

$$
\begin{aligned}
e^{n+1 / 2} & =e^{n-1 / 2}+\Delta \tau C_{0}^{T} h^{n}+\Delta \tau j^{n}, \\
h^{n+1} & =h^{n}-\Delta \tau C_{0} e^{n+1 / 2},
\end{aligned}
$$

where $\Delta \tau=c \Delta t$ is the normalized time step and the upper field indexes introduce the time levels.

On an equidistant mesh, the explicit E/M numerical scheme (6) has a second-order local approximation error in the homogeneous region $O\left(\|\Delta \vec{r}\|^{2}+\Delta \tau^{2}\right), \Delta \vec{r}=$ $(\Delta x, \Delta y, \Delta z)^{T}[10,20,21]$. Applying a Fourier analysis, the following stability condition $[22,23]$ for this numerical scheme can be obtained:

$$
\Delta \tau \leq\left(\frac{1}{\Delta x^{2}}+\frac{1}{\Delta y^{2}}+\frac{1}{\Delta z^{2}}\right)^{-1 / 2} .
$$

This condition is known in the literature as the Courant stability condition.

The scheme (6) is widely used in electromagnetic modeling. However, the E/M algorithm causes nonphysical dispersion of the simulated waves in the computational grid. The phase and group velocities of the discrete waves can differ from the velocity of light by an amount varying with the wavelength, propagation direction, and grid discretization. This leads to additional computational errors known as numerical dispersion errors.

Applying a Fourier analysis, the dispersion relation of the E/M scheme in free space $[20,23,24]$ can be derived:

$$
\frac{\sin ^{2}\left(\frac{\omega}{c} \frac{\Delta \tau}{2}\right)}{\Delta \tau^{2}}=\frac{\sin ^{2}\left(k_{x} \frac{\Delta x}{2}\right)}{\Delta x^{2}}+\frac{\sin ^{2}\left(k_{y} \frac{\Delta y}{2}\right)}{\Delta y^{2}}+\frac{\sin ^{2}\left(k_{z} \frac{\Delta z}{2}\right)}{\Delta z^{2}} .
$$

It can be seen from this relation that, for a homogeneous material, the equidistant mesh $\Delta x=\Delta y=\Delta z$, and the time step $\Delta \tau=\Delta x / \sqrt{3}$, scheme (6) has no dispersion error along the grid diagonals. This property allows for zero dispersion in a desired direction by the rotation of the mesh. However, this approach imposes limitations on the discretization. The only reasonable choice in this case is to 
take equal mesh steps in all three directions. We will not discuss the rotated mesh approach in this paper. In the following we assume that the bunch moves along the $z$ axis.

Let us consider a Gaussian charged bunch of rms length $\sigma$ that moves with the speed of light $c$ in the $z$ direction through a structure of length $L$. The self-field of the ultrarelativistic bunch has only transverse nonzero field components [7-9] and it is like a plane wave. For a plane wave in the $z$ direction, the dispersion relation (8) simplifies to

$$
\frac{\sin \left(\frac{\omega}{c} \frac{\Delta \tau}{2}\right)}{\Delta \tau / 2}=\frac{\sin \left(k \frac{\Delta z}{2}\right)}{\Delta z / 2}
$$

Now assuming that the numerical wave number $k$ differs from the analytical one by $\delta k$, the Taylor expansion to the lowest order of $\delta k$ can be expressed as $[10,25]$

$$
\delta k \approx \frac{1}{24}\left(\frac{\omega}{c}\right)^{3}\left[\Delta z^{2}-\Delta \tau^{2}\right] .
$$

The dispersion error disappears only when the time step is equal to the mesh step $\Delta \tau=\Delta z$. However, this contradicts the stability condition (7). Hence for any stable time step Yee's numerical scheme has a dispersion error in the $z$ direction of the order of

$$
\delta k \approx O\left[\left(\frac{\omega}{c}\right)^{3} \Delta z^{2}\right] .
$$

The Gaussian charged bunch excites electromagnetic fields up to frequencies $\omega \sim c / \sigma$ and for a structure of length $L$ the phase error will be of the order of

$$
L \cdot \delta k \approx O\left(L \sigma^{-3} \Delta z^{2}\right) .
$$

This equation means that Yee's scheme requires a very fine mesh for short bunches and long structures. For an accurate calculation the mesh step should satisfy the following relation:

$$
\Delta z \ll \sigma \sqrt{\frac{\sigma}{L}}
$$

Hence, for the computation of EM fields excited by ultrashort bunches, the development of dispersion-free numerical schemes is an important issue.

\section{B. Implicit FDTD method based on "transverse-electric/transverse-magnetic" splitting of the field components in time}

In this section an implicit TE/TM numerical scheme is discussed [26-28]. For a time step $\Delta \tau$ equal to the longitudinal mesh step $\Delta z$, the TE/TM numerical scheme has no dispersion in the $z$ direction. The time discretization in this scheme is done as follows. On the discrete time axis the "TE" components of the electromagnetic field, $e_{x}, e_{y}, h_{z}$, are defined at the time $\tau=n \Delta \tau$ and the "TM" components of the field, $h_{x}, h_{y}, e_{z}$, at the time $\tau=(n+0.5) \Delta \tau$.

Using the following vector notation for the EM field and the current density components,

$$
u=\left(\begin{array}{c}
h_{x} \\
h_{y} \\
e_{z}
\end{array}\right), \quad v=\left(\begin{array}{c}
e_{x} \\
e_{y} \\
h_{z}
\end{array}\right), \quad j_{u}=\left(\begin{array}{c}
0 \\
0 \\
j_{z}
\end{array}\right), \quad j_{v}=\left(\begin{array}{c}
j_{x} \\
j_{y} \\
0
\end{array}\right) \text {, }
$$

the time-continuous scheme (4) can be rewritten in another matrix form:

$$
\frac{d}{d \tau} u=T_{0} u+L v+j_{u}, \quad \frac{d}{d \tau} v=T_{1} v+L^{T} u+j_{v},
$$

where

$$
\begin{gathered}
T_{0}=\left(\begin{array}{ccc}
0 & 0 & -P_{y}^{0} \\
0 & 0 & P_{x}^{0} \\
\left(P_{y}^{0}\right)^{T} & -\left(P_{x}^{0}\right)^{T} & 0
\end{array}\right), \quad T_{1}=\left(\begin{array}{ccc}
0 & 0 & -\left(P_{y}^{1}\right)^{T} \\
0 & 0 & \left(P_{x}^{1}\right)^{T} \\
P_{y}^{1} & -P_{x}^{1} & 0
\end{array}\right), \\
L=\left(\begin{array}{ccc}
0 & P_{z}^{0} & 0 \\
-P_{z}^{1} & 0 & 0 \\
0 & 0 & 0
\end{array}\right) .
\end{gathered}
$$

With the suggested time discretization the numerical scheme reads

$$
\begin{aligned}
\frac{u^{n+1 / 2}-u^{n-1 / 2}}{\Delta \tau} & =T_{0} \frac{u^{n+1 / 2}+u^{n-1 / 2}}{2}+L v^{n}+j_{u}^{n}, \\
\frac{v^{n+1}-v^{n}}{\Delta \tau} & =T_{1} \frac{v^{n+1}+v^{n}}{2}+L^{T} u^{n+1 / 2}+j_{v}^{n+1 / 2},
\end{aligned}
$$

where the central difference approximation of the derivative in time has been used and a linear interpolation has been applied. The same as Yee's scheme, the TE/TM numerical scheme is a two layer one and can be written as

$$
B \frac{y^{n+1}-y^{n}}{\Delta \tau}+A y^{n}=f^{n},
$$

where we have introduced new matrices and vectors

$$
\begin{aligned}
B & =\left(\begin{array}{cc}
I-\frac{1}{2} \Delta \tau T_{0} & 0 \\
\Delta \tau L^{T} & I-\frac{1}{2} \Delta \tau T_{1}
\end{array}\right), \quad A=\left(\begin{array}{cc}
-T_{0} & -L \\
L^{T} & -T_{1}
\end{array}\right), \\
y^{n} & =\left(\begin{array}{c}
u^{n-1 / 2} \\
v^{n}
\end{array}\right), \quad f^{n}=\left(\begin{array}{c}
j_{u}^{n} \\
j_{v}^{n-1 / 2}
\end{array}\right) .
\end{aligned}
$$

The matrices of Eq. (14) have the following properties:

$$
A=-A^{T}, \quad Q=Q^{T}, \quad \text { with } Q=B-\frac{1}{2} \Delta \tau A .
$$

In paper [28] it was shown that the condition $Q \geq 0$ is necessary and sufficient for the stability of the scheme. This condition can be rewritten in the form

$$
I-\frac{\Delta \tau^{2}}{4} P_{z}^{i}\left(P_{z}^{i}\right)^{T} \geq 0, \quad i=0,1 .
$$


This inequality resembles the well-known stability condition of the explicit FDTD scheme for the one-dimensional electromagnetic problem. The maximal eigenvalue $\lambda_{\max }^{i}$ of the discrete operator $P_{z}^{i}\left(P_{z}^{i}\right)^{T}$ in a staircase approximation of the boundary fulfills the relation

$$
\lambda_{\max }^{i}<4 / \Delta z^{2}, \quad i=0,1
$$

and the stability condition reads

$$
\Delta \tau \leq \Delta z
$$

For azimuthal modes in the rotationally symmetric case, the stability condition has the same form (16) for any azimuthal mode $m$. It is a great advantage of the new scheme compared to Yee's scheme (6) which requires the reduction of the stable time step $[20,29]$ for higher azimuthal modes as

$$
\Delta \tau \leq\left(\left(1.2+0.4 m^{2}\right) \frac{1}{\Delta r^{2}}+1.2 \frac{1}{\Delta z^{2}}\right)^{-1 / 2} .
$$

Following the conventional procedure of stability analyses $[20,22]$, the dispersion relations can be obtained for the TE/TM numerical scheme in the form

$$
\begin{aligned}
\frac{\sin ^{2}\left(\frac{\omega}{c} \frac{\Delta \tau}{2}\right)}{\Delta \tau^{2}}= & \frac{\sin ^{2}\left(k_{z} \frac{\Delta z}{2}\right)}{\Delta z^{2}}+\left(\frac{\sin ^{2}\left(k_{x} \frac{\Delta x}{2}\right)}{\Delta x^{2}}+\frac{\sin ^{2}\left(k_{y} \frac{\Delta y}{2}\right)}{\Delta y^{2}}\right) \\
& \times \cos ^{2}\left(\frac{\omega}{c} \frac{\Delta \tau}{2}\right) .
\end{aligned}
$$

It can be seen from this dispersion equation that, at the "magic" time step $\Delta \tau=\Delta z$, the scheme is dispersion-free in the longitudinal direction. Particularly for equal transverse mesh steps $\Delta x=\Delta y \equiv h$, the dispersion relation for the transverse plane waves $k_{z}=0$ reads as

$$
\tan ^{2}\left(\frac{\omega}{c} \frac{\Delta \tau}{2}\right)=\frac{\Delta \tau^{2}}{h^{2}}\left[\sin ^{2}\left(k_{x} \frac{h}{2}\right)+\sin ^{2}\left(k_{y} \frac{h}{2}\right)\right] .
$$

From the dispersion relation (17) it also follows that Eq. (16) is a necessary stability condition for the implicit TE/TM numerical scheme.

The cancellation of the numerical dispersion error for a particular direction is important in electromagnetic field computation due to the fact that the charged particle beams in accelerators have a well-defined direction of motion (the longitudinal direction). Therefore, the computational domain is very long in the longitudinal direction and relatively short in the transverse plane. Additionally, the electromagnetic field changes very fast in the direction of motion but is relatively smooth in the transverse plane.

Another advantage of the dispersion-free scheme is that for an ultrarelativistic bunch a mesh moving along with the bunch can be used without interpolation errors: the scaled time step is equal to the longitudinal mesh step. The results with the moving mesh for a staircase approximation of the geometry are fully equivalent to the stationary global mesh approach. It follows that for this scheme the mesh step is independent of the structure length $L$ and is related only to the first power of the rms bunch length $\sigma$ [25,26] as $\Delta z<\sigma$.

\section{ONE-DIMENSIONAL NUMERICAL SCHEME FOR FINITE CONDUCTIVITY MODELING}

Let us consider an incident plane EM wave from vacuum onto a conductor surface. Both media are assumed to be of infinite extent (Fig. 3).

For conducting media the relation between the incident angle and the transmitted one can be written in the form of Snell's law [30],

$$
\sin \phi_{t}=\frac{1}{n\left(\phi_{0}, \kappa, \omega\right)} \sin \phi_{0}
$$

where $\phi_{0}$ and $\phi_{t}$ are the incident and the transmitted angles correspondingly, $\omega$ is the frequency of the incident wave. For dielectrics the refraction index $n$ is constant while for conductors [30] it depends on the incident angle, conductivity, and frequency of the incident wave and reads

$n^{2}\left(\phi_{0}, \kappa, \omega\right)=\frac{1}{2}\left[\sin ^{2} \phi_{0}+g\left(\phi_{0}, \kappa, \omega\right) p^{2}\left(1+\xi^{2}\right)+1-\xi^{2}\right]$,

where

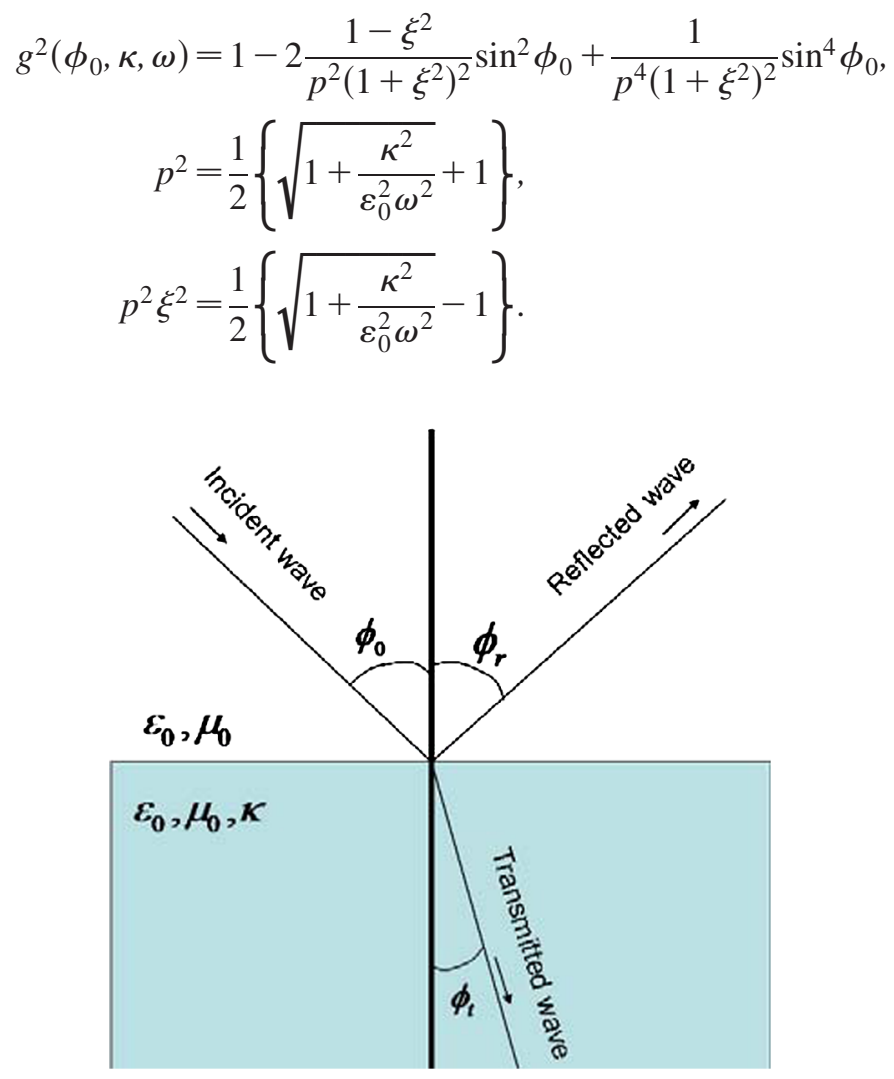

FIG. 3. Transmission and reflection of an EM wave on vacuum-conductor boundary surface. 
Here one should keep in mind that $p$ and $\xi$ are functions of frequency and conductivity.

For good conductors with $\kappa \geq 10^{4} \mathrm{~S} / \mathrm{m}$, the following condition is usually fulfilled:

$$
\frac{\kappa}{\varepsilon_{0} \omega} \gg 1
$$

Under this condition the parameters $p$ and $\xi$ in Eq. (19) can be approximated as

$$
p^{2} \rightarrow \frac{1}{2} \frac{\kappa}{\varepsilon \omega}, \quad \xi \rightarrow 1 .
$$

Using these expressions it is easy to show that the refraction index is much larger than unity $n\left(\phi_{0}, \kappa, \omega\right) \gg 1$. Thus, when condition (20) is fulfilled, the incident waves are transmitted perpendicular to the boundary surface $\left(\phi_{t} \sim 0\right)$, i.e., only the tangential components of the electric and magnetic fields survive in the conducting media.

In modern accelerators the vacuum chambers are usually made of highly conducting materials like stainless steel, aluminum, or copper and the condition (20) is satisfied. As an example, for a stainless steel vacuum chamber $\left(\kappa=1.4 \times 10^{6} \Omega^{-1} \mathrm{~m}^{-1}\right)$ and a Gaussian bunch with rms length of $1 \mu \mathrm{m}$, one has $\kappa /\left(\varepsilon_{0} \omega\right) \approx 500$.

Thus, for highly conducting media only the tangential component of the wave in the resistive region (the transmitted wave) needs be accounted for and the task is reduced to a one-dimensional electromagnetic problem in the metal. The so-called 1D conducting line model can be applied locally instead of the full three-dimensional set of Maxwell's equations.

The propagation of the tangential field in the conductor is described by the 1D electromagnetic problem. The line wire is discretized as shown in Fig. 4.

The excitation source of the EM field in the conducting media is the tangential magnetic field at the boundary cell. We use an implicit 1D numerical scheme,

$$
\begin{aligned}
& e_{c}^{n+1}=A e_{c}^{n}+B P_{c} \frac{h_{c}^{n+1}+h_{c}^{n}}{2}, \\
& h_{c}^{n+1}=h_{c}^{n}+\Delta \tau P_{c}^{T} \frac{e_{c}^{n+1}+e_{c}^{n}}{2},
\end{aligned}
$$

where

$$
e_{c}^{n}=\left(\begin{array}{c}
e_{c, 0}^{n} \\
\vdots \\
e_{c, N}^{n}
\end{array}\right), \quad h_{c}^{n}=\left(\begin{array}{c}
h_{c,-(1 / 2)}^{n} \\
\vdots \\
h_{c, N-(1 / 2)}^{n}
\end{array}\right)
$$

The banded matrix $P_{c}$ plays the role of a discrete differential operator and the index $c$ is used to note that the EM fields are considered in a conductive region. The matrices $A$ and $B$ are diagonal with entries

$$
\begin{gathered}
a_{00}=e^{-0.5 \kappa Z_{0} \Delta \tau}, \quad b_{00}=2 \frac{1-a_{00}}{\kappa Z_{0}}, \quad a_{i i}=e^{-\kappa Z_{0} \Delta \tau}, \\
b_{i i}=\frac{1-a_{i i}}{\kappa Z_{0}}, \quad i=1, \ldots, N .
\end{gathered}
$$

The boundary conditions at the interface are given by

$$
e_{c, N}^{n}=0, \quad h_{c, 0}^{n}=h_{c, 0}^{n+1}=h_{\tau}^{n+0.5},
$$

where $h_{\tau}^{n+0.5}$ is the tangential component of the magnetic field in the vacuum cell to which the wire belongs.

\section{NEW HYBRID TE/TM IMPLICIT NUMERICAL SCHEME FOR FINITE CONDUCTIVITY MODELING}

In this section a hybrid TE/TM implicit numerical scheme for finite conductivity modeling in azimuthally symmetric accelerating structures is introduced [31-33]. It is assumed that the condition (20) is fulfilled.

The hybrid scheme uses the TE/TM splitting numerical scheme for the vacuum part of the geometry. The boundary cells of the grid in vacuum region are supplied with onedimensional line wires to model the wave propagation in the metal. In this model the tangential electric field components at the interface are used to update the magnetic field components in the boundary cells of the vacuum region. At the same time, the excitation source for the 1D problem in metal is the tangential component of the magnetic field at the interface. To describe the tangential components of the electromagnetic fields at the interface, a local coordinate system $\{p, \varphi, s\}$ is introduced as shown in Fig. 5.

It can be seen from Fig. 5 that the $s$ axis of the local coordinate system is always directed along the normal

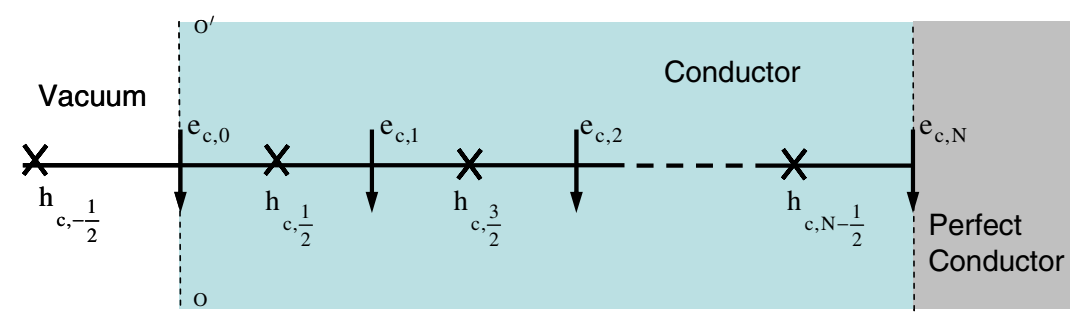

FIG. 4. The line wire discretization in the conductor region. 


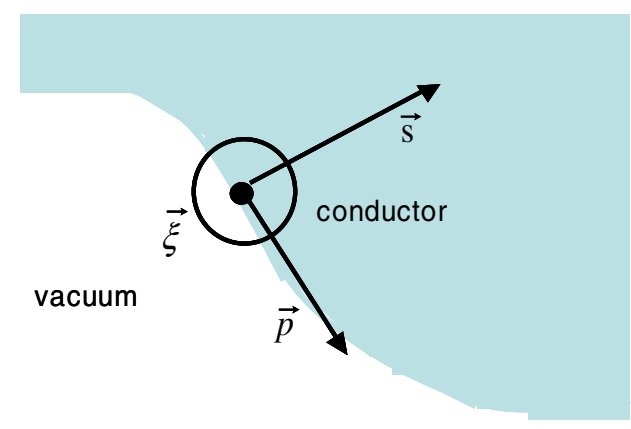

FIG. 5. Boundary local coordinate system.

vector to the surface. Thus, according to the previous section the EM field components that are tangential to the surface will propagate into the conductive region in the direction of the $s$ axis.

In this work the boundary interface is considered in a staircase approximation [34,35]. It results in $O(1)$ local approximation error near the interface while the 3D numerical scheme in free space introduced in Sec. IV has second-order accuracy [26]. Thus, due to the interface $O(1)$ local approximation error, the global accuracy of the 3D numerical scheme is reduced to the first order. The second order convergence of the scheme can be recovered by conformal methods that have been already implemented for perfect electric boundary condition (PEC) problems [29]. The first numerical experiments with a direct application of the conformal method to the hybrid scheme resulted in serious instabilities and a rigorous study is necessary to improve the stability. Thus, the hybrid scheme will be introduced here only for the staircase approximation of the interface.

The TE/TM splitting hybrid numerical scheme is realized for electromagnetic problems with rotationally symmetric geometries. A cylindrical coordinate $\{r, \varphi, z\}$ system is used, where $z$ is the longitudinal coordinate. The rotational symmetry of the electromagnetic problem allows one to use an azimuthal expansion and to write the explicit dependence of the EM fields on the azimuthal coordinate $\varphi[8]$ :

$$
\begin{aligned}
& \left\{E_{r}, E_{z}, H_{\varphi}\right\}_{m}(r, \varphi, z, t)=\left\{E_{r}, E_{z}, H_{\varphi}\right\}_{m}(r, z, t) \cos (m \varphi), \\
& \left\{H_{r}, H_{z}, E_{\varphi}\right\}_{m}(r, \varphi, z, t)=\left\{H_{r}, H_{z}, E_{\varphi}\right\}_{m}(r, z, t) \sin (m \varphi),
\end{aligned}
$$

where $m$ is the azimuthal mode number.

In accelerators the principal source of current is the charged particle beam. For a bunch moving at the speed of light $c$ with transverse offset $a$ parallel to the axis of a rotationally symmetric structure, the bunch current $\vec{j}_{b}$ can be represented via multipole expansion as

$$
\vec{j}_{b}=\frac{\vec{c} \lambda(z / c-t) \delta(r-a)}{\pi a} \sum_{m=0}^{\infty} \frac{\cos (m \varphi)}{1+\delta_{m, 0}}
$$

with $\delta_{m, 0}= \begin{cases}1, & m=0 \\ 0, & m \neq 0,\end{cases}$

where $\lambda(z / c-t)$ is the longitudinal charge distribution and $\delta(r-a)$ is the Dirac delta function.

Hence, the three-dimensional problem is reduced to a set of independent two-dimensional problems and the space discretization is required only in two directions $\{r, z\}$.

Usually the accelerating structures are supplied with ingoing and outgoing beam pipes. In our considerations, the structures will be supplied with perfectly conducting pipes. This allows one to use an analytical representation to define the initial electromagnetic fields in the ingoing pipe and to use the indirect integration method for the wake potential calculations [36] in the outgoing pipe.

The boundary cells of the grid, which are in contact with the conductive region, require a careful consideration. The goal is to model the conductive boundary in time domain. The thickness of the modeled conductor is taken to be much larger than the skin depth of the metal. The tangential components of the magnetic field in the boundary cells of the vacuum region will be used as the excitation sources for the 1D electromagnetic problems in the conductive region. These magnetic field components are tangential to the boundary surface and perpendicular to the tangential electric fields. Thus, the modeling of the conductive boundary in the time domain is reduced to solution of $1 \mathrm{D}$ electromagnetic problems in the metal.

According to Fig. 5, the boundary conditions at the interfaces for the $1 \mathrm{D}$ electromagnetic problem are given

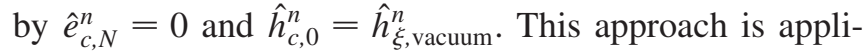
cable when the thickness of the modeling conductor is taken to be much larger than the skin depth of the metal in the considered problem.

From the multipole expansion of the fields (23) it follows that for the monopole case $(m=0)$ the nonzero field components are $E_{r}, E_{z}$, and $H_{\varphi}$. Thus, the monopole case will be considered separately from the higher-order multipole case.

\section{A. Monopole case $(m=0)$}

The discretization of the problem is graphically illustrated in Fig. 6, where the 1D conductive lines for finite conductivity modeling are shown.

The magnetic field component $\hat{h}_{\varphi_{i, j}}$ in cell $\mathbf{A}$ (Fig. 6) is the excitation source for the $1 \mathrm{D}$ electromagnetic problems in the transverse and the longitudinal directions, i.e., these 1D problems are equivalent. Thus, the electric field components tangential to the boundary are equal at any time step $\hat{e}_{c}^{n} \equiv \hat{e}_{c r}^{n}=\hat{e}_{c z}^{n}$. This implies that for each mesh cell the number of 1D problems is reduced from two to one. The TE/TM numerical scheme for monopole case in original form reads 


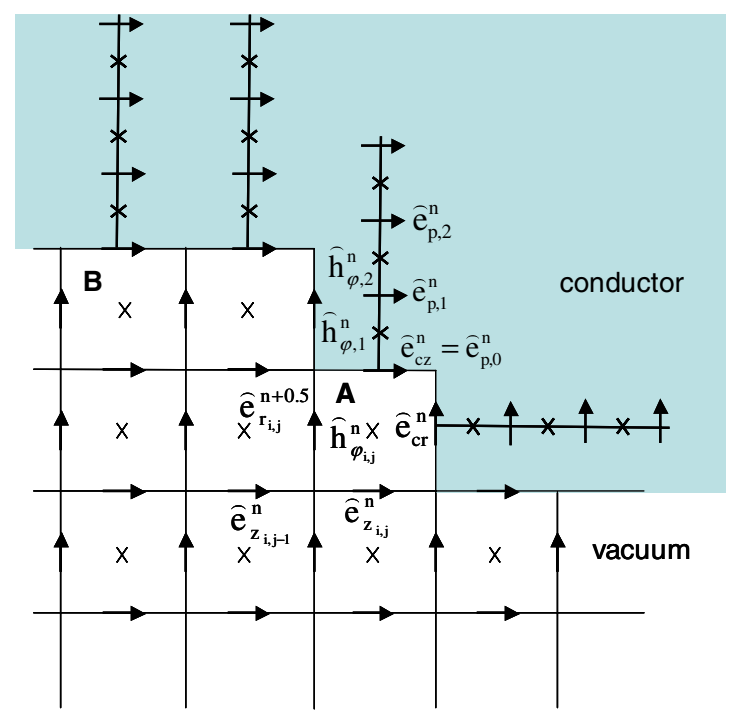

FIG. 6. The grid in the vacuum with $1 \mathrm{D}$ conductive lines in the metal (monopole case).

\section{TE component}

$\hat{e}_{r}^{n+1 / 2}=\hat{e}_{r}^{n-1 / 2}-\Delta \tau M_{\varepsilon_{r}^{-1}} P_{z}^{T} \hat{h}_{\varphi}^{n}$,

TM components

$\hat{h}_{\varphi}^{\#} \equiv \hat{h}_{\varphi}^{n}+\frac{\Delta \tau}{2} M_{\mu_{\varphi}^{-1}}\left[P_{z} \hat{e}_{r}^{n+1 / 2}-P_{r} \hat{e}_{z}^{n}+l_{c} \hat{e}_{c p}^{n}\right]$,

$\hat{e}_{z}^{n+1}=\hat{e}_{z}^{n}+\Delta \tau \Psi_{0}^{-1} M_{\varepsilon_{z}^{-1}}\left[P_{r}^{T} \hat{h}_{\varphi}^{\#}-\hat{j}_{z}^{n}\right]$,

$1 \mathrm{D}$ updates in conductive region,

$\hat{h}_{\varphi}^{n+1}=\hat{h}_{\varphi}^{\#}+\frac{\Delta \tau}{2} M_{\mu_{\varphi}^{-1}}\left[P_{z} \hat{e}_{r}^{n+1 / 2}-P_{r} \hat{e}_{z}^{n+1}+l_{c} \hat{e}_{c p}^{n+1}\right]$,

with the three-diagonal matrix

$$
\Psi_{0} \equiv\left(I+\frac{\Delta \tau^{2}}{4} M_{\varepsilon_{z}^{-1}} P_{r}^{T} M_{\mu_{\varphi}^{-1}} P_{r}+\frac{\Delta \tau^{2}}{4} M_{\varepsilon_{z}^{-1}} M_{\mu_{r}^{-1}}\right),
$$

where $I$ is the unit diagonal matrix, $\hat{e}_{c p}^{n}, \hat{e}_{c p}^{n+1}$ are the voltages at the conductive surface, and $l_{c}$ is the conductive edge length. The conductive edge length for A-like cells (Fig. 6) is $l_{c}=2$, and for $\mathbf{B}$-like cells is $l_{c}=1$. In the staircase approximation of the interface the material matrices $M_{\mu^{-1}}, M_{\varepsilon^{-1}}$ are diagonal and contain geometric information about the sizes of edges and facets.

\section{B. Higher-order multipole case $(\boldsymbol{m}>0)$}

For the higher-order multipole case, all six components of the field need to be taken into account. The components $\hat{e}_{r}^{n}, \hat{e}_{z}^{n}, \hat{h}_{\varphi}^{n}$ of the EM field are graphically shown in Fig. 6. The other three components $\hat{h}_{r}^{n}, \hat{h}_{z}^{n}, \hat{e}_{\varphi}^{n}$ of the field are illustrated in Fig. 7. For the case of higher-order multipoles, a second electric field component $\hat{e}_{c \varphi}^{n}$ tangential to the boundary interface exists. In order to find the field component $\hat{e}_{c \varphi}^{n}$, the second 1D problem in the conductor

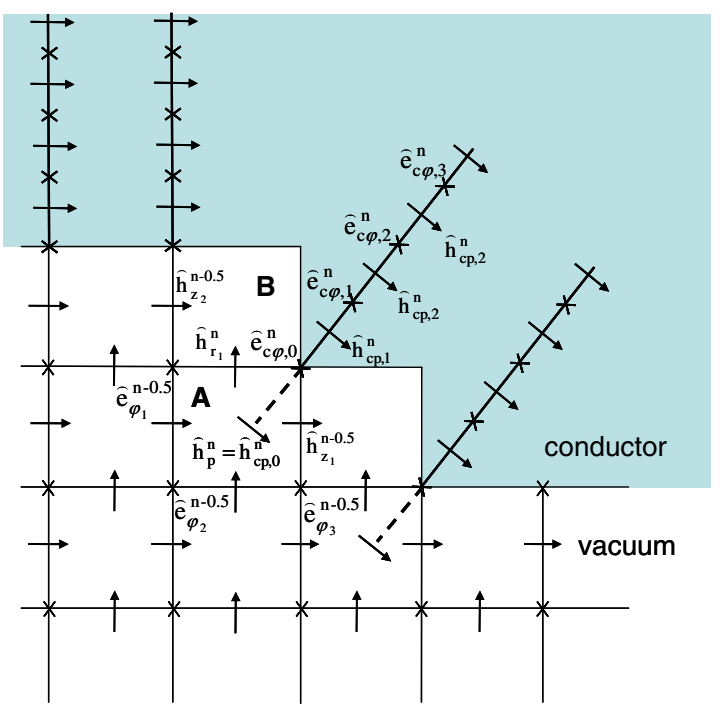

FIG. 7. Two-dimensional view of the cylindrical mesh of the vacuum region.

should be solved. As an excitation source for this 1D problem, the $\hat{h}_{p}^{n}$ (Fig. 7) magnetic field from the boundary cell in vacuum is taken. For A-like cells (Fig. 7) this magnetic field vector is defined as a sum of the magnetic field vectors $\hat{h}_{r}^{n}$ and $\hat{h}_{z}^{n}$ projected onto the cell diagonal (Fig. 8). Following the notation illustrated in Fig. 8, the $\hat{h}_{p}^{n}$ field can be written as

$$
\hat{h}_{p}^{n}=\hat{h}_{z_{1}}^{n} \cos \alpha+\hat{h}_{r_{1}}^{n} \sin \alpha, \quad \operatorname{tg} \alpha=\frac{\Delta r}{\Delta z} .
$$

For B-like cells (Fig. 7), the $\hat{h}_{p}^{n}$ field is equal to $\hat{h}_{z}^{n}$.

Note that in the TE/TM numerical scheme the longitudinal component of the magnetic field $\hat{h}_{z}$ on the discrete time axis is defined at the time level $(n+0.5)$. Thus, this field at the time level $n$ can be found by linear interpolation.

Thus, for modeling of the conductive boundary in the time domain, one should solve two 1D EM problems for each vacuum boundary cell, and the hybrid numerical scheme for an azimuthal mode number $m$ reads

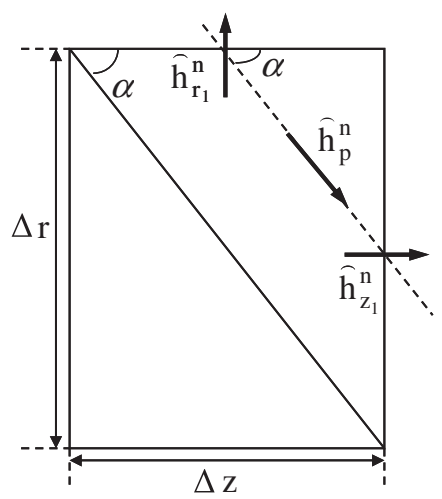

FIG. 8. The discrete magnetic field components in the grid cell. 
TE components

$$
\begin{aligned}
\hat{e}_{\varphi}^{\#} & \equiv \hat{e}_{\varphi}^{n-1 / 2}+\frac{\Delta \tau}{2} M_{\varepsilon_{\varphi}^{-1}}\left[P_{z}^{T} \hat{h}_{r}^{n}-P_{r}^{T} \hat{h}_{z}^{n-1 / 2}\right], \\
\hat{e}_{r}^{\#} & \equiv \hat{e}_{r}^{n-1 / 2}+\frac{\Delta \tau}{2} M_{\varepsilon_{r}^{-1}}\left[m \hat{h}_{z}^{n-1 / 2}-P_{z}^{T} \hat{h}_{\varphi}^{n}\right], \\
\hat{h}_{z}^{n+1 / 2} & =\hat{h}_{z}^{n-1 / 2}+\Delta \tau \Psi_{h}^{-1} M_{\mu_{z}^{-1}}\left[P_{r} \hat{e}_{\varphi}^{\#}-m \hat{e}_{r}^{\#}+\hat{e}_{c \varphi}^{n}\right], \\
\hat{e}_{\varphi}^{n+1 / 2} & =\hat{e}_{\varphi}^{\#}+\frac{\Delta \tau}{2} M_{\varepsilon_{\varphi}^{-1}}\left[P_{z}^{T} \hat{h}_{r}^{n}-P_{r}^{T} \hat{h}_{z}^{n+1 / 2}\right], \\
\hat{e}_{r}^{n+1 / 2} & =\hat{e}_{r}^{\#}+\frac{\Delta \tau}{2} M_{\varepsilon_{r}^{-1}}\left[m \hat{h}_{z}^{n+1 / 2}-P_{z}^{T} \hat{h}_{\varphi}^{n}\right],
\end{aligned}
$$

TM components

$$
\begin{aligned}
\hat{h}_{\varphi}^{\#} & \equiv \hat{h}_{\varphi}^{n}+\frac{\Delta \tau}{2} M_{\mu_{\varphi}^{-1}}\left[P_{z} \hat{e}_{r}^{n+1 / 2}-P_{r} \hat{e}_{z}^{n}+l_{c} \hat{e}_{c p}^{n}\right], \\
\hat{h}_{r}^{\#} & \equiv \hat{h}_{r}^{n}+\frac{\Delta \tau}{2} M_{\mu_{r}^{-1}}\left[m \hat{e}_{z}^{n}-P_{z} \hat{e}_{\varphi}^{n+1 / 2}+\hat{e}_{c \varphi}^{n}\right], \\
\hat{e}_{z}^{n+1} & =\hat{e}_{z}^{n}+\Delta \tau \Psi_{e}^{-1} M_{\varepsilon_{z}^{-1}}\left[P_{r}^{T} \hat{h}_{\varphi}^{\#}-m \hat{h}_{r}^{\#}-\hat{j}_{z}^{n}\right],
\end{aligned}
$$

two 1D updates in conductive region,

$$
\begin{aligned}
& \hat{h}_{\varphi}^{n+1}=\hat{h}_{\varphi}^{\#}+\frac{\Delta \tau}{2} M_{\mu_{\varphi}^{-1}}\left[P_{z} \hat{e}_{r}^{n+1 / 2}-P_{r} \hat{e}_{z}^{n+1}+l_{c} \hat{e}_{c p}^{n+1}\right], \\
& \hat{h}_{r}^{n+1}=\hat{h}_{r}^{\#}+\frac{\Delta \tau}{2} M_{\mu_{r}^{-1}}\left[m \hat{e}_{z}^{n+1}-P_{z} \hat{e}_{\varphi}^{n+1 / 2}+\hat{e}_{c \varphi}^{n+1}\right],
\end{aligned}
$$

where the operators

$$
\begin{aligned}
& \Psi_{e} \equiv\left(I+\frac{\Delta \tau^{2}}{4} M_{\varepsilon_{z}^{-1}} P_{r}^{T} M_{\mu_{\varphi}^{-1}} P_{r}+\frac{\Delta \tau^{2}}{4} m^{2} M_{\varepsilon_{z}^{-1}} M_{\mu_{r}^{-1}}\right), \\
& \Psi_{h} \equiv\left(I+\frac{\Delta \tau^{2}}{4} M_{\mu_{z}^{-1}} P_{r} M_{\varepsilon_{\varphi}^{-1}} P_{r}^{T}+\frac{\Delta \tau^{2}}{4} m^{2} M_{\mu_{z}^{-1}} M_{\varepsilon_{r}^{-1}}\right)
\end{aligned}
$$

are three-diagonal matrices and the equations can be solved easily using the "sweep" method.

The hybrid implicit numerical scheme is designed in a way that the stability condition (16) of the original TE/TM scheme in the vacuum region is preserved. Thus, at the magic time step $\Delta \tau=\Delta z$ the hybrid scheme described here is dispersion free in the longitudinal direction.

\section{CONVERGENCE OF THE HYBRID SCHEME}

The convergence study of the hybrid numerical scheme has been performed for the case of a round pipe with finite conductive walls. We compare the numerical results with the steady-state analytical solution. For a point charge moving in an infinitely long round pipe of radius $b$ with the offset $a$ parallel to the symmetry axis, the analytical steady-state solution for the longitudinal wake potential of the $m$ th multipole is well known [37] and can be written as

$$
w_{/ /, m}(s)=q_{m} r^{m} \cos (m \varphi) F_{m}(s),
$$

where $q_{m}$ is the $m$ th multipole charge moment

$$
q_{m}=\frac{(m+1)}{\pi} \frac{a^{m}}{b^{2(m+1)}}
$$

and the function $F_{m}(s)$ is given as

$$
\begin{aligned}
F_{m}(s)= & -\frac{4}{\varepsilon_{0}}\left[\frac{1}{3} e^{-\eta^{2 / 3}\left(s / s_{0}\right)} \cos \left(\sqrt{3} \eta^{2 / 3} \frac{s}{s_{0}}\right)\right. \\
& \left.-\frac{\sqrt{2}}{\pi} \eta \int_{0}^{\infty} \frac{x^{2}}{x^{6}+8 \eta^{2}} e^{-x^{2}\left(s / s_{0}\right)} d x\right] .
\end{aligned}
$$

Here $\eta=\left(m+1+\delta_{m, 0}\right) / 2, \delta_{m, 0}$ is the Kronecker delta function, and $s_{0}$ is so-called characteristic distance given as

$$
s_{0}=\left(\frac{2 c b^{2} \varepsilon_{0}}{\kappa}\right)^{1 / 3} .
$$

For the bunch distribution $\rho(s)$, the longitudinal wake potential is given by the convolution

$$
W_{\|, m}^{\rho}(s)=\int_{-\infty}^{s} w_{\|, m}\left(s-s^{\prime}\right) \rho\left(s^{\prime}\right) d s^{\prime}
$$

The transverse wake potential $W_{\perp, m}^{\rho}$ can be obtained through the Panowsky-Wenzel theorem [38]. The wake potentials are given per unit length of the pipe.

The convergence of the hybrid scheme has been analyzed for a Gaussian bunch of rms length $\sigma=1 \mathrm{~mm}$ moving through a round pipe of radius $a=1 \mathrm{~cm}$ and wall conductivity $\kappa=10^{5} \mathrm{~S} / \mathrm{m}$. The results of the convergence study are presented in Fig. 9. The convergence of
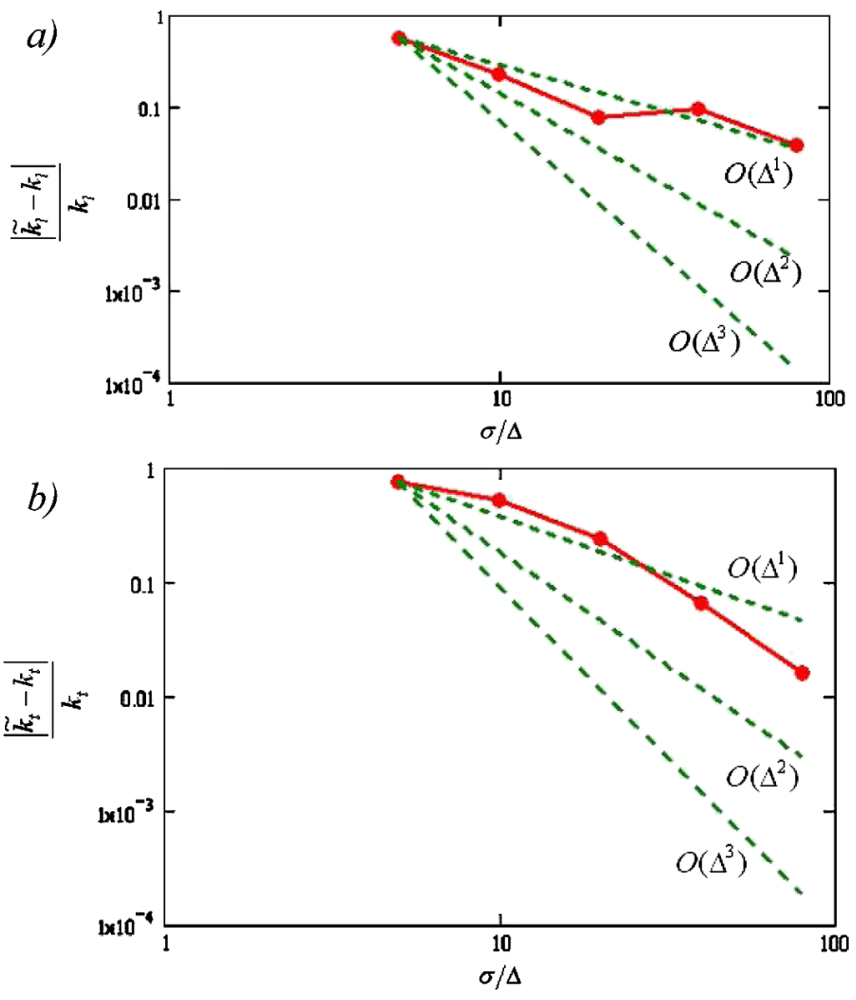

FIG. 9. Convergence (logarithmic scale) of the monopole loss and dipole kick factors (red) and predictions up to the 3rd order (green dashed). 
the numerically calculated loss and kick factors to the analytical values $k_{l}=1.31 \mathrm{~V} / \mathrm{pC}$ and $k_{t}=75.5 \mathrm{~V} / \mathrm{pC} / \mathrm{m}$, respectively, can be seen. In these plots the horizontal axis corresponds to the number of mesh points on an rms bunch length $\sigma$ and $\Delta$ is the space step. All calculations in this and the next sections are done with an equidistance mesh and a magic time step $\Delta \tau=\Delta z=\Delta r$. It can be seen that the numerical scheme is accurate to first order. For the convergence study the geometrical mesh has been shifted from the boundary interface. Otherwise a second-order convergence will be observed. Similar tests of the convergence of the hybrid scheme have been performed for higher-order harmonics and similar results have been observed.

\section{NUMERICAL TESTS ON ROTATIONALLY SYMMETRIC STRUCTURES}

In this section several examples of the wake potential calculations in various resistive structures are presented. The results are compared with known analytical solutions and with the results obtained by other numerical methods. We consider the following structures: an infinitely long resistive tube, a resistive insert, and a resistive tapered collimator.

\section{A. Steady-state resistive wake potentials}

The first test is the calculation of the steady-state longitudinal and transverse short-range wake potentials of a Gaussian bunch with rms length $\sigma=1 \mathrm{~mm}$ moving through a round pipe of radius $a=1 \mathrm{~cm}$ with wall conductivity $\kappa=10^{5} \mathrm{~S} / \mathrm{m}$. For this simple geometry, the alignment of the geometric mesh with the boundary interface is possible.

Figure 10 shows the comparison of the numerically calculated longitudinal monopole (a) and transverse dipole (b) wake potentials with analytical wakes. The numerical results are given for a mesh resolution of 10 points on the rms bunch length $\sigma$. In this case (where the mesh coincides with the boundary interface), the error in the loss and kick factors is about $3 \%$ whereas to obtain the same accuracy in the staircase approximation (where the mesh is shifted from the boundary interface) more than 40 points on $\sigma$ is required.

\section{B. The wake potentials for a resistive insert}

The second test is the calculation of the longitudinal and transverse wake potentials of the Gaussian bunch passing through a finite-length resistive cylinder supplied with infinitely long perfectly conducting beam pipes (Fig. 11). The resistive part of the cylinder has radius $a=1 \mathrm{~cm}$, length $g=10 \mathrm{~cm}$, and conductivity $\kappa=10^{4} \mathrm{~S} / \mathrm{m}$.

The analytical solutions for the monopole loss $k_{l}$ and dipole kick $k_{t}$ factors of the Gaussian bunch in this structure are given in [39]
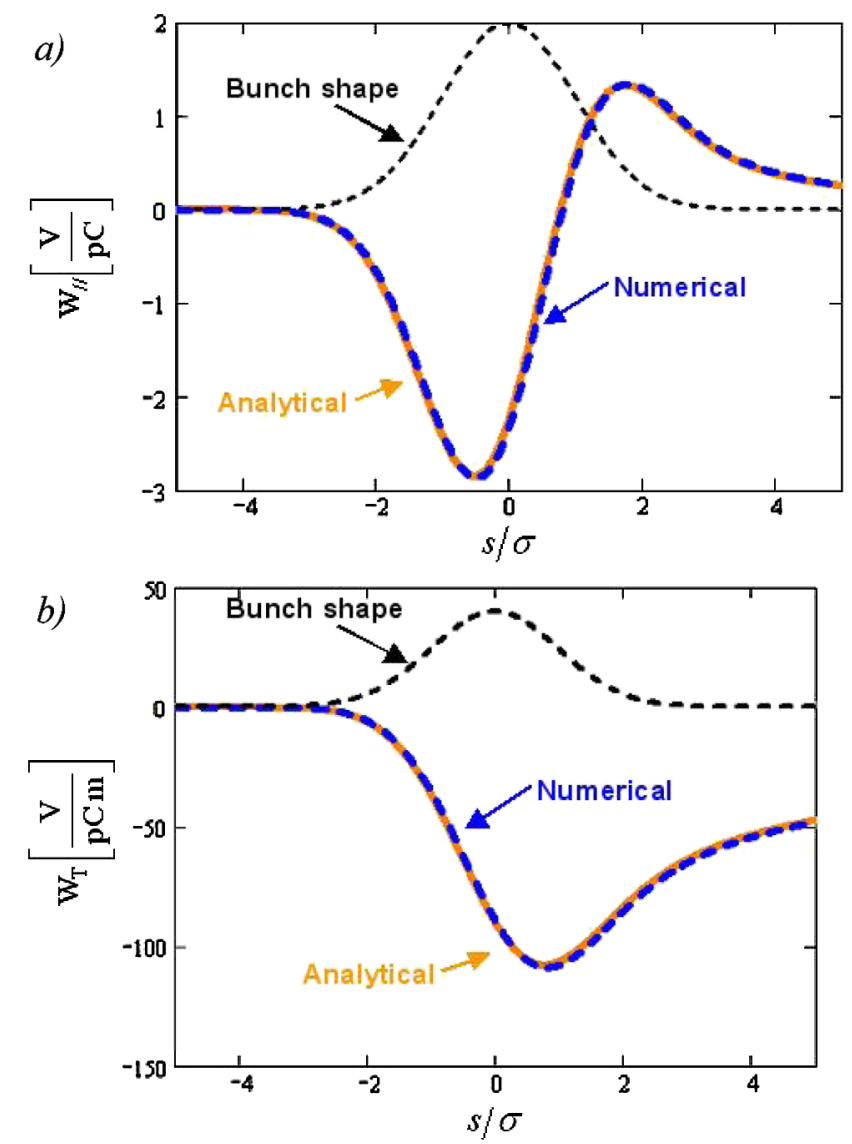

FIG. 10. Comparison of the numerical longitudinal monopole and transverse dipole wake potentials (blue dashed) with analytical solutions (orange solid).

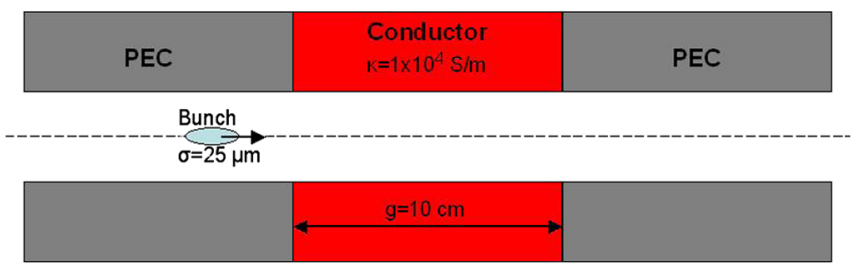

FIG. 11. Cross section of the finite-length resistive cylinder between infinitely long perfectly conducting pipes.

$$
\begin{aligned}
k_{l} & =\frac{c Z_{0} g}{2 \pi^{2} a \sqrt{2 \kappa Z_{0} s_{g}^{3 / 2}}} K_{l}\left(\frac{\sigma}{s_{g}}\right), \\
k_{t} & =\frac{c Z_{0} \sqrt{g s_{g}}}{\pi^{2} a^{3}} K_{t}\left(\frac{\sigma}{s_{g}}\right), \\
s_{g} & =\sqrt{\frac{g}{2 Z_{0} \kappa}}
\end{aligned}
$$

where the functions $K_{l}$ and $K_{t}$ are 


$$
\begin{aligned}
K_{l}(u)= & \int_{0}^{\infty} d x x^{-3 / 2} e^{-x^{2} u^{2}}\left[1-e^{-x^{2}}+\frac{2 x}{\sqrt{\pi}}-e^{-x^{2}} \operatorname{erfi}(x)\right], \\
K_{t}(u)= & \int_{0}^{\infty} d x x^{-5 / 2} e^{-x^{2} u^{2}} \\
& \times \operatorname{erfi}(u x)\left[1-e^{-x^{2}}+\frac{2 x}{\sqrt{\pi}}-e^{-x^{2}} \operatorname{erfi}(x)\right] .
\end{aligned}
$$

Figure 12 presents the numerically obtained longitudinal monopole and transverse dipole wake potentials, and the analytical steady-state wakes $[7,8]$ for a Gaussian bunch of rms length $\sigma=25 \mu \mathrm{m}$.

The numerically obtained loss and kick factors are $58 \mathrm{~V} / \mathrm{pC}$ and $42.6 \mathrm{~V} / \mathrm{pC} / \mathrm{m}$, respectively. The numerical results coincide with the analytical prediction (29) for the loss factor $(57 \mathrm{~V} / \mathrm{pC})$ and the kick factor $(41.5 \mathrm{~V} / \mathrm{pC} / \mathrm{m})$ with accuracies of $1.7 \%$ and $2.3 \%$, respectively.

In the demonstrated example of a $10 \mathrm{~cm}$-long resistive insert, it is clearly seen that the steady-state loss $(16 \mathrm{~V} / \mathrm{pC})$ and kick $(9.6 \mathrm{~V} / \mathrm{pC} / \mathrm{m})$ factors underestimate the exact results by $\sim 75 \%$.
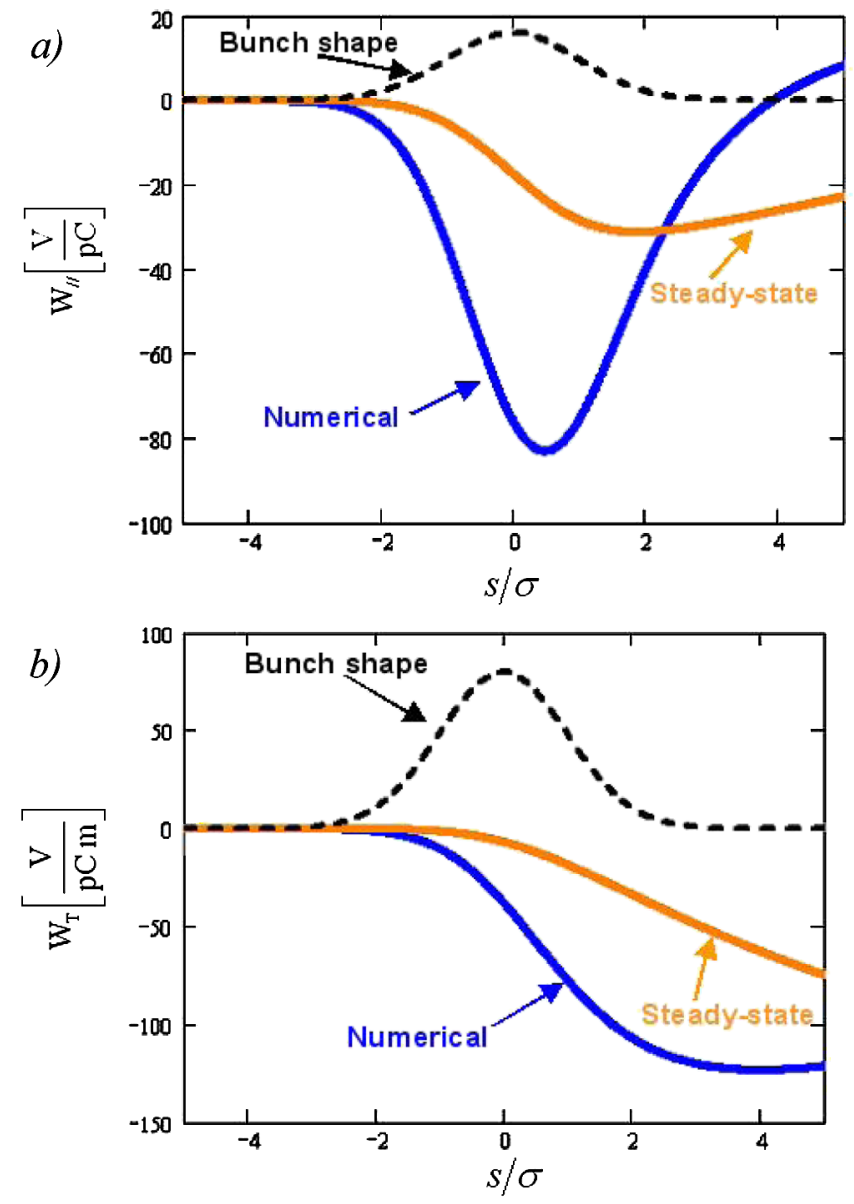

FIG. 12. Comparison of transient (blue solid) and steady state (orange solid) wake potentials.

\section{Wake potentials in a rotationally symmetric tapered collimator}

The third example is a rotationally symmetric tapered collimator (Fig. 13) with parameters $d=10 \mathrm{~mm}, b=$ $6 \mathrm{~mm}, L_{1}=200 \mathrm{~mm}, L_{2}=100 \mathrm{~mm}$, and conductivity $\kappa=10^{4} \mathrm{~S} / \mathrm{m}$.

The comparison of the longitudinal monopole wake potentials obtained for the tapered collimator with finite (full) and perfect (geometrical) conducting walls is presented in Fig. 14. The numerically obtained loss factor for the Gaussian bunch with rms length $\sigma=50 \mu \mathrm{m}$ is $270 \mathrm{~V} / \mathrm{pC}$. This value is 2 times larger than the loss factor for the same structure with perfectly conducting walls $(133 \mathrm{~V} / \mathrm{pC})$. Hence, the real wake cannot be obtained as a direct sum of the geometric and the steady-state solutions. A similar situation is observed for the transverse wake potential.

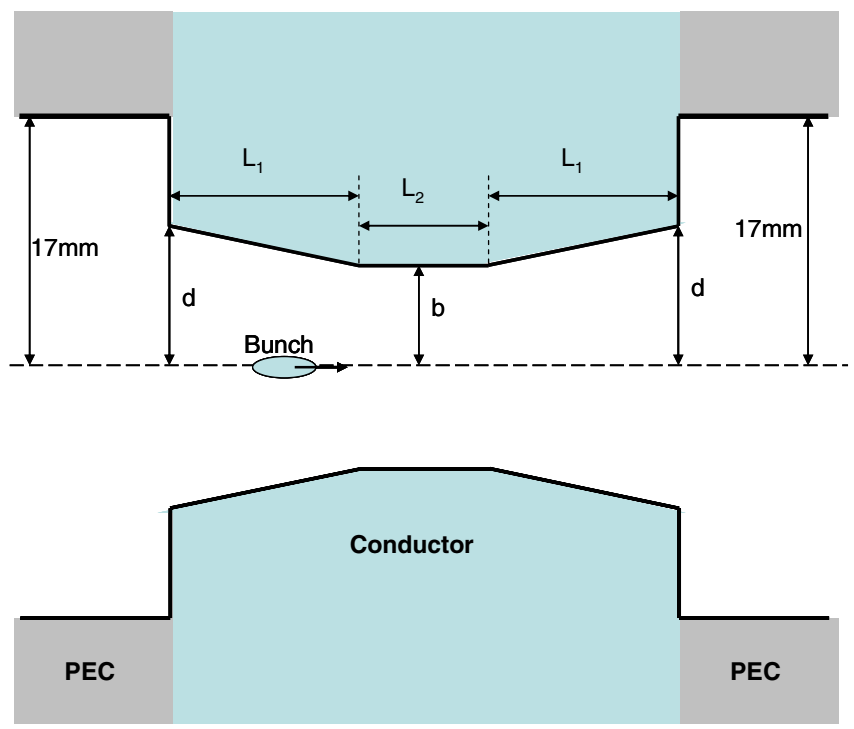

FIG. 13. Cross section of the tapered collimator.

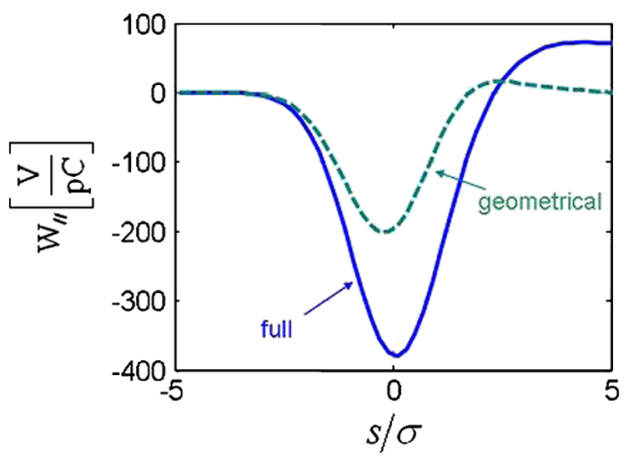

FIG. 14. Longitudinal wake potentials for the tapered collimator with finite (blue solid) and perfect (green dashed) conducting walls. 

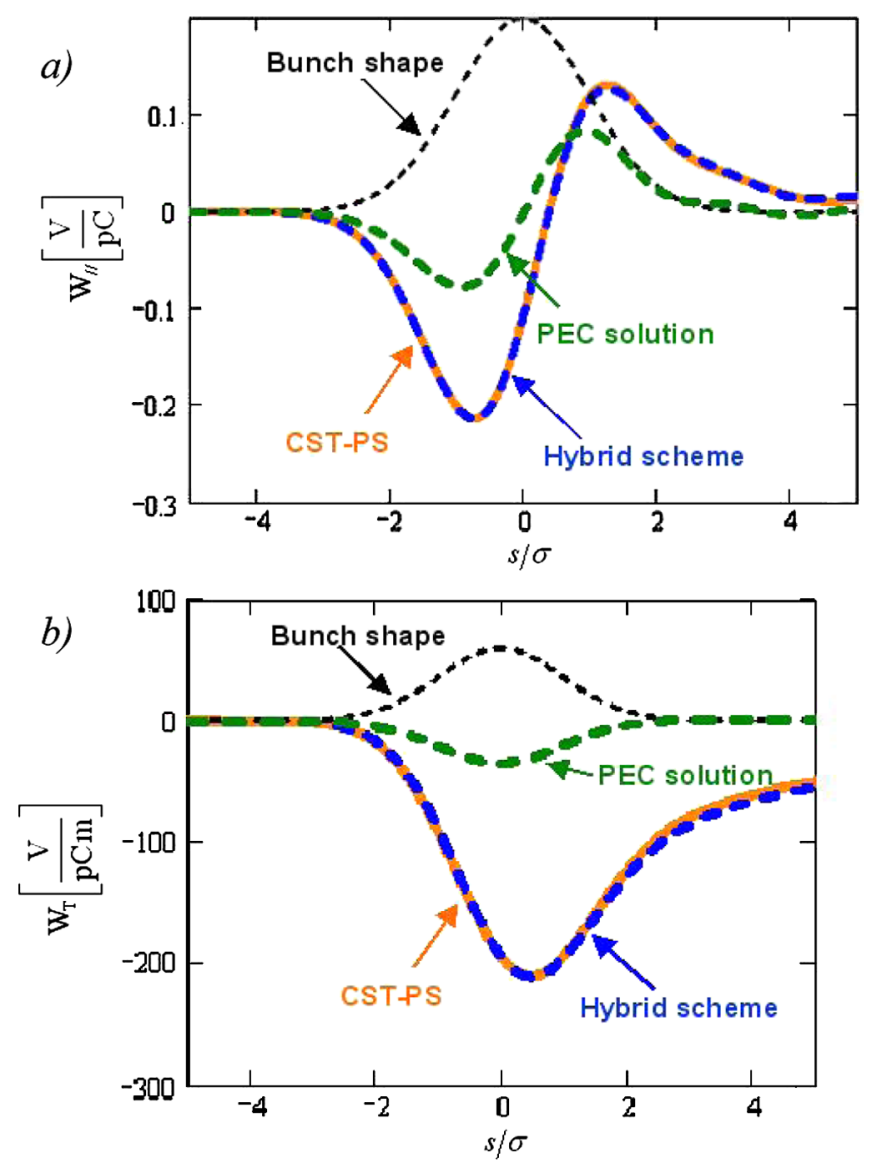

FIG. 15. Comparison of longitudinal wake potential calculated by CST PARTICLE STUDIO (orange solid) and using the hybrid scheme (blue dashed).

In the last example the wake potentials computed by the hybrid numerical scheme and by CST MICROWAVE STUDIO are compared. This commercial software uses Yee's finite difference scheme. In order to obtain accurate results with CST MICROWAVE STUDIO, the Gaussian bunch is taken to be quite long, $\sigma=20 \mathrm{~mm}$, and the tapered collimator (Fig. 13) is taken to be shorter with the parameters $d=$ $10 \mathrm{~mm}, b=6 \mathrm{~mm}, L_{1}=L_{2}=30 \mathrm{~mm}$, and low conductivity $\kappa=100 \mathrm{~S} / \mathrm{m}$. Note that the conductivity was reduced to obtain a larger wake potential than that in the PEC case.

Figure 15 presents the comparison of the longitudinal monopole and transverse dipole wake potentials obtained by CST PARTICLE STUDIO (CST-PS) and by the new hybrid numerical code. The wake potentials calculated with the new code are obtained with 40 mesh points per rms bunch length $\sigma$. They are in good agreement with the results obtained by CST-PS.

\section{CONCLUSION}

In this paper a new hybrid numerical scheme for wakefield calculations in rotationally symmetric structures is introduced. This hybrid scheme is developed for wakefields excited by ultrashort bunches moving through the structures with walls of finite high conductivity. This scheme is dispersion free in the longitudinal direction (in the vacuum region), which allows one to calculate the wakefields for very long $(L>1 \mathrm{~m})$ structures without loss of accuracy. The realization of the scheme is done for a staircase approximation of the boundaries which reduces the calculation accuracy to first order. Several tests have been performed to evaluate the accuracy of the hybrid scheme. The results are in good agreement both with analytical and numerical results.

\section{ACKNOWLEDGMENTS}

We thank M. Lomperski for useful discussions and corrections.

[1] K. Kubo, A. Seryi, N. Walker, and A. Wolski, ICFA Beam Dyn. Newslett. 44, 13 (2007).

[2] J. Arthur et al., LCLS Design Study Report No. SLAC-R521, 1998.

[3] M. Altarelli et al., DESY Technical Design Report No. 2006-097, 2006.

[4] A. W. Chao, Physics of Collective Beam Instabilities in High Energy Accelerators (Wiley, New York, 1993).

[5] B. W. Zotter and S. A. Kheifetz, Impedances and Wakes in High-Energy Particle Accelerators (World Scientific, Singapore, 1997).

[6] T. Weiland and B. Zotter, Part. Accel. 11, 143 (1981).

[7] H. Henke and O. Napoly, in Proceedings of the Second European Particle Accelerator Conference (Editions Frontiáeres, Nice, 1990), pp. 1046-1048.

[8] S. Ratschow and T. Weiland, Phys. Rev. ST Accel. Beams 5, 052001 (2002).

[9] R.W. Hockney and J.W. Eastwood, Computer Simulation Using Particles (IOP Publishing Ltd., Bristol, 1988).

[10] K. S. Yee, IEEE Trans. Antennas Propag. 14, 302 (1966).

[11] T. Weiland, in Proceedings of the 11th International Conference on High-Energy Accelerators, Geneva, Switzerland (Birkhauser, Geneva, Switzerland, 1980), pp. 570-575.

[12] MAFIA Collaboration, MAFIA manual, CST GmbH, Darmstadt, 1997.

[13] T. Weiland, IEEE Trans. Nucl. Sci. 30, 2489 (1983).

[14] A. D. Greenwood, K. L. Cartwright, J. W. Luginsland, and E. A. Baca, J. Comput. Phys. 201, 665 (2004).

[15] CST MICROWAVE STUDIO is a trademark of CST GmbH, Bad Nauheimer Str. 19, 64289 Darmstadt, Germany [http://www.cst.com.

[16] I. Zagorodnov and T. Weiland, Phys. Rev. ST Accel. Beams 8, 042001 (2005).

[17] E. Gjonaj, T. Lau, T. Weiland, and R. Wanzenberg, ICFA Beam Dyn. Newslett. 45, 38 (2008).

[18] T. Weiland, Int. J. Numer. Model. 9, 295 (1996).

[19] T. Weiland, Part. Accel. 17, 227 (1985). 
[20] Computational Electrodynamics: The Finite-Difference Time-Domain Method, edited by A. Taflove and S.C. Hagness (Artech House, London, 2000).

[21] A. A. Samarskii, The Theory of Difference Schemes (Marcel Dekker, New York, 2001).

[22] K. S Kunz and R. J. Luebbers, The Finite Difference Time Domain Method for Electromagnetism (CRC Press, New York, 1993).

[23] M. Levy, Parabolic Equation Methods for Electromagnetic Wave Propagation (Cornwall, England, 2000).

[24] S. Wang and F. L. Teixeira, IEEE Trans. Antennas Propag. 51, 1818 (2003).

[25] M. Dohlus and I. Zagorodnov, J. Comput. Phys. 228, 2822 (2009).

[26] I. Zagorodnov and T. Weiland, J. Comput. Phys. 207, 69 (2005).

[27] I. Zagorodnov and T. Weiland, in Proceeding of the ICAP 2002 (World Scientific, East Lansing, 2002).

[28] I. Zagorodnov, R. Schumann, and T. Weiland, J. Comput. Phys. 191, 525 (2003).

[29] I. Zagorodnov, R. Schuhmann, and T. Weiland, Int. J. Numer. Model. 16, 127 (2003).

[30] M. Born and E. Wolf, Principles of Optics (Pergamon Press, London, 1980).
[31] A. Tsakanian, Ph.D. thesis, Hamburg University [Report No. DESY-THESIS-2010-041, Hamburg, 2010].

[32] A. Tsakanian, M. Dohlus, and I. Zagorodnov, in Proceedings of the 11th European Particle Accelerator Conference, Genoa, 2008 (EPS-AG, Genoa, Italy, 2008), TUPP075 [http://accelconf.web.cern.ch/AccelConf/e08/ papers/tupp075.pdf].

[33] A. Tsakanian, M. Dohlus, and I. Zagorodnov, in Proceedings of the 23rd Particle Accelerator Conference, Vancouver, Canada, 2009 (IEEE, Piscataway, NJ, 2009), TH5PFP059 [http://epaper.kek.jp/PAC2009/papers/ th5pfp059.pdf].

[34] A. C. Cangellaris and D. B. Wright, IEEE Trans. Antennas Propag. 39, 1518 (1991).

[35] J. B. Schneider and K. L. Shlager, IEEE Trans. Antennas Propag. 45, 1830 (1997).

[36] I. Zagorodnov, Phys. Rev. ST Accel. Beams 9, 102002 (2006).

[37] K. L. F. Bane, SLAC Report No. SLAC-AP-87, 1991.

[38] W. K. H. Panowsky and W. A. Wenzel, Rev. Sci. Instrum. 27, 947 (1956).

[39] S. Krinsky, B. Podobedov, and R. L. Gluckstern, Phys. Rev. ST Accel. Beams 7, 114401 (2004). 